\title{
When in doubt should we cut it out? The role of surgery in non-small cell lung cancer
}

\section{J-E C Holty, M K Gould}

\section{Will we ever really know whether surgery is effective in patients with resectable NSCLC?}

$\mathrm{D}$ spite being a preventable disease the public health impact of lung cancer is daunting. Lung cancer accounts for more than an estimated one million deaths each year. ${ }^{1}$ Unfortunately, most persons with nonsmall-cell lung cancer (NSCLC) have unresectable disease at presentation with an overall 5 year survival rate of approximately $15 \% .^{23}$ In contrast, 99\% of patients with prostate cancer, $88 \%$ of those with breast cancer and $63 \%$ of patients with colon cancer are alive at 5 years. ${ }^{3}$ For early stage NSCLC the preferred treatment is surgical resection, with an estimated 75000 procedures performed in the US and 3000 in the UK each year. ${ }^{4}$ This preference is supported by favourable 5 year survival rates for patients with potentially resectable tumours (stage IA 67\%, stage IB, 57\%, stage IIA 55\%, stage IIB 39\%). ${ }^{5}$ However, up to $30 \%$ of patients with stage I and $65 \%$ of patients with stage II cancers will experience recurrence within 5 years of resection. ${ }^{6}$ Furthermore, although recent survival rates for resected stage I and II disease have increased compared with historical controls, ${ }^{7}$ this has been attributed in part to more careful surgical lymph node analysis (stage migration) $)^{8-10}$ and the detection of earlier disease (lead time bias). ${ }^{1}$

Surgical resection of lung cancer with intent to cure was introduced in the early 1930s by Graham and Singer. ${ }^{12}$ Surgery was quickly accepted as the preferred treatment modality for early stage NSCLC, based in large part on the results from observational and retrospective studies. ${ }^{13}{ }^{14}$ However, relying on these type of studies can be misleading because the apparent benefit of surgical resection may be due to patient characteristics, supportive measures and care, or other unknown confounding factors.

In the early 1960s Morrison and colleagues were among the first to evaluate surgical resection of lung cancer in a randomised controlled trial. ${ }^{15}$ This relatively small but often cited study randomised 58 patients with lung cancer to surgery versus radiotherapy. The surgery group was younger $(40 \% \mathrm{v}$ $32 \%$ under 55 years of age) and had less mediastinal lymph node involvement $(30 \% v 36 \%)$. Although the overall 4 year survival rate was better in the surgical arm (23\% v 7\%), this improvement did not reach statistical significance. Subgroup analysis of patients with squamous cell carcinoma did show a statistically significant improved survival benefit with surgery (30\% v 6\%; $\mathrm{p}<0.05)$. However, the surgical arm had substantially higher 1 year mortality (57\% v 36\%). Of the 30 patients randomised to surgery, $43 \%$ did not receive radical resection primarily due to inoperable lesions found at thoracotomy. This suggests that many randomised patients did not have early stage disease. Furthermore, 11 of the patients randomised to surgery crossed over to the radiotherapy arm. Given these important limitations, it is difficult to establish whether the benefits of surgical resection exceed the risks for early stage NSCLC based on this study alone.

Some have questioned the therapeutic validity of surgery due to the lack of evidence from large randomised trials. ${ }^{14}$ In formulating their recommendation in support of surgery for medically fit patients with stage I disease, the Lung Cancer Guidelines Panel of the American College of Chest Physicians concluded that the level of evidence was fair. ${ }^{16}$ In contrast, the British Thoracic Society $^{17}$ concluded that curative surgical resection in patients with stage I or II disease was supported only by expert opinion. $^{18}$ Given the importance of determining the actual survival benefit of surgical resection, the systematic review reported by Wright et al ${ }^{19}$ in this issue of Thorax is the first to provide a comprehensive assessment of the therapeutic efficacy of surgery for localised NSCLC. However, despite an exhaustive literature search, none of the 11 included randomised trials had a control group that did not receive surgery. Thus, the study authors were unable to directly assess the efficacy of surgery, and the sobering conclusion is that, while we may believe that surgery is an effective treatment, the evidence is scant. This is unfortunate, given that many patients are more risk averse to surgery than is realised by most physicians, ${ }^{20}$ and that the goal of surgery is to improve not only survival but also postoperative quality of life..$^{21} 22$

Although surgical resection is the clinical standard of care for local stage disease, controversy still exists regarding the optimal surgical technique. In order to clarify this controversy, Wright and colleagues ${ }^{19}$ identified studies that compared various surgical approaches. For example, the authors identified three randomised trials that assessed the survival benefit of radical lymphadenectomy compared with lymph node sampling at resection. ${ }^{23-25}$ Individually, these three studies showed no statistically significant survival benefit for patients receiving complete mediastinal lymph node dissection. However, Wright and colleagues showed that the pooled hazard for overall mortality in patients receiving systematic nodal dissection was significantly reduced $(0.78 ; \mathrm{p}=0.005)$.

Unfortunately, this conclusion is tempered by the considerable evidence of study design bias in these three studies. In two of the studies ${ }^{23} 25$ an intention-totreat analysis could not be performed due to post-randomisation exclusions, a bias that tends to favour the treatment arm especially in the setting of a metaanalysis. ${ }^{26}$ Specifically, some of the patients were excluded due to upstaging following radical lymphadenectomy (stage migration). ${ }^{8-10}$ Stage specific survival benefits due to stage migration during complete lymph node dissection have been observed in randomised trials of both gastric ${ }^{27}$ and breast cancer ${ }^{28}$ patients. Furthermore, two of the three studies $^{24} 25$ were unblinded during follow up assessment which may have resulted in a detection bias favouring the intervention. ${ }^{29}$ Forthcoming results from a recently completed large randomised controlled trial conducted by the American College of Surgeons Oncology Group should provide more definitive evidence. $^{30}$

It is surprising that, despite the enormous public health impact of lung cancer, only 14 articles describing 11 randomised trials met the inclusion criteria following an extensive literature search. Some of the included studies did not report the mode of allocation concealment and none had a clear intention-to-treat analysis. These two study design biases are known to increase the magnitude of a treatment's effectiveness. $^{29}$ None of the included studies 
evaluated quality of life, performance status, or costs. The assessment of quality of life measures is now fundamental in the evaluation of lung cancer treatment outcomes..$^{21} 2231$

Given the dearth of patients studied and apparent study design weaknesses in most trials, is it possible to infer whether surgery is beneficial? For example, the only study that compared surgery alone with a non-surgical intervention (radiotherapy) had inconclusive results. ${ }^{15}$ In another study that enrolled patients initially assessed as inoperable but who were operable after radiotherapy, no significant difference in disease free or overall survival was found between the surgical and non-surgical control groups, although significant respiratory complications were noted with surgery. ${ }^{32}$ In the one study that compared limited resection to lobectomy, only a non-significant statistical trend towards improved overall survival in the lobectomy group was shown. ${ }^{3}$ However, the lobectomy group also trended towards more surgical complications. Given the lack of indirect evidence of a surgical benefit, one might quibble with the assertion by Wright and colleagues that "by inference, some surgery might be better than no surgery". Better designed and appropriately powered randomised trials are needed to establish definitively whether radical lymphadenectomy (versus lymph node sampling) or lobectomy (versus limited resection) are the preferred surgical techniques.

One must therefore ask whether we will ever really know whether surgery is effective in patients with resectable NSCLC. In the absence of well designed randomised trials, one might be tempted to rely on the results of observational studies to assess the efficacy of surgical resection for local stage disease. However the key element that differentiates observational from randomised controlled trials is the potential for selection bias. Unfortunately, measures to control for selection biases in observational studies of surgery have not been sufficient. For example, a retrospective study in patients with stage I disease compared 291 surgically treated patients with 45 patients who did not undergo surgery and found substantially higher 5 year survival rates in the patients who underwent surgical resection $(70 \% \vee 10 \%) .{ }^{34}$ However, the patients who underwent surgery were younger and had less medical co-morbidity, two confounding factors that were not specifically controlled for. Future observational trials should incorporate propensity scores or other sophisticated statistical methods to deal with overt bias and use appropriate sensitivity analysis to deal with potential hidden biases.
The preferred moral basis for performing a randomised controlled trial is clinical equipoise. Equipoise requires that genuine uncertainty exists throughout the medical profession with regard to the best treatment strategy. ${ }^{35}$ Because most clinicians believe that local stage disease is potentially curable with surgery, designing a randomised trial that includes an untreated control arm is highly problematic and probably unethical. ${ }^{13}$ However, equipoise for performing a trial of surgery versus no treatment may exist in certain limited circumstances-for example, in elderly lung cancer patients who present with relatively small ground glass opacities. $^{36}{ }^{37}$ Another way of achieving equipoise is a "preference trial" in which only those patients who are indifferent in their preference for treatments-that is, surgery $v$ radiation, or surgery $v$ best supportive care-are randomised..$^{38}$ Currently, up to $30 \%$ of patients with early stage disease are either not offered or choose not to undergo surgery. ${ }^{39}$ In the future it should be a priority to perform studies (when clinical equipoise exits) that compare traditional surgery with newer less invasive techniques such as stereotactic radiation and radiofrequency ablation. At the very least, we owe it to our patients to discover which surgical and minimally invasive techniques have risk to benefit ratios that are most favourable.

Thorax 2006;61:554-556

doi: $10.1136 /$ thx.2006.060558

\section{Authors' affiliations}

J-E C Holty, M K Gould, Division of

Pulmonary and Critical Care Medicine,

Stanford University Medical Center, Stanford, CA, USA

M K Gould, VA Palo Alto Health Care System, Palo Alto, CA, USA J-E C Holty, M K Gould, Center for Primary Care and Outcomes Research, Stanford University, Stanford, CA, USA

Correspondence to: $\mathrm{Dr}$ J-E C Holty, Division of Pulmonary and Critical Care Medicine, Stanford University School of Medicine, 300 Pasteur Drive, H3143, Stanford, CA 943055236, USA; iholty@stanford.edu

Drs Holty and Gould have no financial conflict of interest or competing interests to disclose.

\section{REFERENCES}

1 Parkin DM, Bray F, Ferlay J, et al. Global cancer statistics. CA Cancer J Clin 2005;55:74-108.

2 Ries LAG. Influence of extent of disease, histology and demographic factors on lung cancer surviva in the SEER population-based data. Semin Surg Oncol 1994; 10:21-30.

3 Jemal A, Murray T, Ward E, et al. Cance statistics, 2005. CA Cancer J Clin 2005; 55: 10-30.

4 Sedrakyan A, van der Meulen J, O'Byrne K, et al. Postoperative chemotherapy for non-small cell lung cancer: a systematic review and meta-analysis. J Thorac Cardiovasc Surg 2004; 128:414-9.

5 Mountain CF. Revisions in the international system for staging lung cancer (special reports). Chest 1997;111:1710-7.

6 lascone C, DeMeester TR, Albertucci M, et al Local recurrence of resectable non-small-cell lung carcinoma of the lung. Cancer 1986;57:471-6.

7 Wada H, Tanaka F, Yanagihara K, et al. Time trends and survival after operations for primary lung cancer from 1976 through 1990. J Thorac Cardiovasc Surg 1996;112:349-55.

8 Testa T, Nahum MA, Spinelli E, et al. The 'Will Rogers Effect' on stage grading. Ann Ital Chir 1999:70:829-30.

9 Padilla J, Calvo V, Penalver JC, et al. Stage I nonsmall cell lung cancer up to $3 \mathrm{~cm}$ in diameter: prognostic factors. Arch Bronconeumol 2004:40: $110-3$

10 Feinstein AR, Sosin DM, Wells CK. The Will Rogers phenomenon. Stage migration and new diagnostic techniques as a source of misleading statistics for survival in cancer. N Engl J Med 1985;312:1604-8.

11 Yoshino I, Baba H, Fukuyama S, et al. A time trend of profile and surgical results in 1123 patients with non-small cell lung cancer. Surgery 2002;131:S242-8.

12 Graham EA, Singer JJ. Successful removal of an entire lung for carcinoma of the bronchus. JAMA 1933;101:1371-4.

13 Geddes DM. The natural history of lung cancer: a review based on the rates of tumour growth. $\mathrm{Br} J$ Dis Chest 1979;73:1-17.

14 Lederle FA, Niewoehner DE. Lung cancer surgery. A critical review of the evidence. Arch Intern Med 1994;154:2397-400.

15 Morrison R, Deeley TJ, Cleland WP. The treatment of carcinoma of the bronchus: a clinical trial to compare surgery and supervoltage radiotherapy. Lancet 1963;1:683-4.

16 Smythe WR. Treatment of stage I non-small cell lung carcinoma. Chest 2003; 123:181-7S.

17 British Thoracic Society. Lung cancer guidelines. Available at http://www.brit-thoracic.org.uk (accessed 21 March 2006)

18 National Institute for Health, Clinical Excellence (NICE). Clinical guideline 24: Lung cancer. February 2005. Available at http:// www.nice.org/uk (accessed 21 March 2006).

19 Wright G, Manser RL, Byrnes G, et al. Surgery for non-small cell lung cancer: systematic review and meta-analysis of randomised controlled trials. Thorax 2006:61:597-603.

20 Mazur DJ, Hickam DH. Five-year survival curves: how much data are enough for patient-physician decision making in general surgery. Eur J Surg 1996;162:101-4

21 Handy JR, Asaph JW, Skokan L, et al. What happens to patients undergoing lung cancer surgery? Outcomes and quality of life before and after surgery. Chest 2002;122:21-30.

22 Yoshimura H. Quality of life (QOL) versus curability for lung cancer surgery. Ann Thorac Cardiovasc Surg 2001;7:127-32.

23 Izbicki J, Passlick B, Pantel K, et al. Effectiveness of radical systematic mediastinal lymphadenectomy in patients with resectable nonsmall cell lung caner: results of a prospective randomized trial. Ann Surg 1998;227:138-44

24 Sugi K, Nawata K, Fujita N, et al. Systematic lymph node dissection for clinically diagnosed peripheral non-small-cell lung cancer less than $2 \mathrm{~cm}$ in diameter. World J Surg 1998;22:290-5.

25 Wu YL, Huang ZF, Wang SY, et al. A randomized trial of systematic nodal dissection in resectable non-small cell lung cancer. Lung Cancer 2002;36: 1-6

26 Tierney JF, Stewart LA. Investigating patient exclusion bias in meta-analysis. Int $J$ Epidemiol 2005; 34:79-87.

27 Wu CW, Hsiung CA, Lo SS, et al. Stage migration influences on stage-specific survival comparison between D1 and D3 gastric cancer surgeries. EJSO 2005;31:153-7.

28 Galimberti V, Veronesi P, Arnone P, et al. Stage migration after biopsy of internal mammary chain lymph nodes in breast cancer patients. Ann Surg Oncol 2002;9:924-8 
29 Schulz KF, Chalmers I, Hayes RJ, et al. Empirical evidence of bias: dimensions of methodological quality associated with estimates of treatment effects in controlled trials. JAMA 1995;273:408-12.

30 Allen MS, Darling GE, Pechet TTV, et al. Morbidity and mortality of major pulmonary resections in patients with early-stage lung cancer: initial results of the randomized prospective ACOSOG Z0030 trial. Ann Thorac Surg 2006;81:1013-20.

31 Mohan A, Guleria R, Pathak AK, et al. Quality of life measures in lung cancer. Indian $J$ Cancer 2005;42:125-32
32 Warram J. Preoperative irradiation of cancer of the lung: final report of a therapeutic trial (a collaborative study). Cancer 1975;36:914-25.

33 Ginsberg R, Rubenstein LV. Randomized trial of lobectomy versus limited resection for $\mathrm{T} 1 \mathrm{NO}$ nonsmall cell lung cancer. Ann Thorac Surg 1995:60:615-23.

34 Flehinger BJ, Kimmel M, Melamed MR. The effect of surgical treatment on survival from early lung cancer. Implications for screening. Chest 1992:101:1013-8.

35 Freedman B. Equipoise and the ethics of clinical research. N Engl J Med 1987;317:141-5.
36 Yankelevitz DF, Reeves AP, Kostis WJ, et al. Small pulmonary nodules: volumetrically determined growth rates based on CT evaluation. Radiology 2000;215(Suppl):607-9

37 Kodama K, Higashiyama M, Yokouchi $\mathrm{H}$, et al. Natural history of pure ground-glass opacity after long-term follow-up of more than 2 years. Ann Thorac Surg 2002;73:386-92.

38 Torgerson DJ, Sibbald B. Understanding controlled trials. What is a patient preference trial? BMJ 1998:316:360.

39 Bach PB, Cramer LD, Warren JL, et al. Racial differences in the treatment of early stage lung cancer. N Engl J Med 1999;341:1198-205.

\section{Diagnosing CF: sweat, blood and years J S Elborn, J M Bradley}

\section{Use of algorithms for the diagnosis of CF}

$\mathrm{T}$ he diagnosis of cystic fibrosis (CF) is usually straightforward. The accepted criteria for the diagnosis of CF is one phenotypic characteristic of $\mathrm{CF}$ (such as lung disease or pancreatic malabsorption), or a positive neonatal screening result, or a positive history of $\mathrm{CF}$ in a sibling plus a raised sweat chloride level, positive nasal potential difference $(\mathrm{PD})$ test, or two mutations in the CFTR gene. ${ }^{\prime}$ In countries with neonatal screening the diagnosis is made in most cases using either an immunoreactive trypsinogen (IRT) test on a heel prick blood sample or direct detection of genetic mutations. ${ }^{2}$ Missed cases (false negatives) from screening are almost all pancreatic sufficient with minimal lung disease, and may have a consequent delay in diagnosis. ${ }^{2}$ In countries which do not yet have neonatal screening for $\mathrm{CF}$, most children present in the first year of life with failure to thrive, recurrent respiratory infections, or both. For such children a sweat test is the most important investigation to confirm the diagnosis. Some patients also present with clinical disease later in childhood and into adult life and diagnosis can be more difficult for a number of reasons.

In late diagnosed patients there is a wider range of presenting phenotypes in addition to the common presentations with respiratory infection and pancreatic malabsorption. ${ }^{3}$ Some present with single organ pulmonary disease (bronchiectasis), pancreatitis, severe sinusitis, or infertility. The explanation for this is that some mutations of the CFTR gene are associated with an atypical phenotype, usually with less severe lung disease. Over 1000 mutations of the CFTR gene have now been described, but only a proportion are associated with disease. ${ }^{4}$

Mutations of the CFTR gene which cause disease can be classified as follows: class 1, defective protein synthesis (e.g. G542X); class 2, defective protein processing (e.g. $\Delta \mathrm{F} 508$ ); class 3 , defective protein regulation (e.g. G551D). These three classes are considered to be severe mutations and are associated with the classic CF phenotype. Class 4 (e.g. R117H) and class 5 (e.g. $3849+10 \mathrm{kbC} \rightarrow \mathrm{T}$ and IVS8-5T) are associated with altered chloride conductance of CFTR or reduced expression and with mild phenotypes. There are, in addition, a number of CFTR polymorphisms associated with mild phenotypes, particularly the number of TG repeats in IVS8. ${ }^{4}$

At the most mild phenotypic extreme are people with congenital bilateral absence of the vas deferens (CBAVD) who have one or two mutations of the CFTR gene. The vas deferens is the most sensitive organ to CFTR dysfunction. About $50 \%$ of men who present with infertility and who have CBAVD have one or two mutations of the CFTR gene. ${ }^{56}$ They may have a sweat chloride level of $30-60 \mathrm{mmol} / \mathrm{l}$ and a mildly abnormal nasal PD. For these individuals the pulmonary prognosis is almost certainly very good, although some may develop mild sinopulmonary symptoms related to the CF phenotype later in life. It is important to consider carefully the most appropriate diagnostic label for such individuals. This applies to other atypical presentations associated with CF such as idiopathic pancreatitis, bronchiectasis, heat exhaustion, allergic bronchopulmonary aspergillosis (ABPA), and chronic sinusitis, as all of these presentations can cause diagnostic dilemmas. ${ }^{2} 67$

In this issue of Thorax De Boeck et al present two consensus algorithms from a Diagnostic Working Group of European experts on the diagnosis of $\mathrm{CF}$ and review the supportive diagnostic tests such as sweat testing, genotyping, nasal PD and measurements of intestinal currents. These algorithms provide a helpful approach to making the diagnosis of CF. The two algorithms can be used following clinical suspicion of the diagnosis of CF or neonatal screening. They lead to diagnostic classification of CF as classic or non-classic, or exclusion of the diagnosis of CF. A further small group of mildly affected patients might be considered as having a CFTR related disorder. This is usually single organ disease such as CBAVD, recurrent pancreatitis, or severe sinusitis. Such patients may have a single mutation of their CFTR gene and a normal or slightly increased sweat chloride concentration. Single organ disease such as this should not result in a diagnosis of $\mathrm{CF}$, either classic or non-classic, and may be classified according to a WHO diagnostic list, though this is not an exhaustive list of diagnoses. The algorithms will also help confidently to exclude a diagnosis of CF.

These algorithms are likely to be helpful in addressing the question: "Is this disease CF?" For example, in patients attending a general respiratory clinic (paediatric or adult) with symptoms or signs suggesting bronchiectasis, the diagnosis of CF should be considered. Patients with bronchiectasis who have a history of symptoms starting in childhood should all have a sweat test and the diagnostic algorithm used from there. In particular, patients from whom typical organisms of CF such as Staphylococcus aureus, Pseudomonas aeruginosa, or a member of the Burkholderia genus are isolated should have a sweat test to determine if their disease is related to CFTR dysfunction. The sweat test is the most straightforward first investigation in such patients and then the first diagnostic algorithm should be followed as appropriate. Confirmatory genotyping can then be undertaken to 